\title{
Angina Pós-Cirurgia de Revascularização Miocárdica Secundária à Estenose de Artéria Subclávia Esquerda. Causa Incomum, Tratamento Comum
}

\author{
Rogério Sarmento-Leite, Luis Maria Yordi, Gilberto Heineck, Paulo Ortiz Jr., Carlos A. M. Gottschall
}

Porto Alegre, RS

Estenose da artéria subclávia ocorre em aproximadamente $3 \%$ da população com doença aterosclerótica. O maior fator preditivo de sua ocorrência é a presença de doença vascular periférica, e os sintomas podem ser bastante limitantes. A terapêutica percutânea tem se constituído no tratamento de eleição para estes casos, e com o advento dos stents tornou-se um método eficaz, seguro e com altas taxas de sucesso. Um homem de 54 anos com passado de cirurgia de revascularização miocárdica (CRM) pontes de safena para ramos marginal (Mg) e diagonal (Dg) e implante de artéria mamária interna esquerda (MIE) para artéria descendente anterior (ADA), hipertensão arterial sistêmica, dislipidemia, tabagismo e doença vascular periférica foi internado por angina classe IV. No exame físico apresentava diminuição de pulsos em membros inferiores e diferencial de pressão de $20 \mathrm{mmHg}$ nos membros superiores. O eletrocardiograma mostrava isquemia subendocárdica em parede anterior. A cineangiocoronariografia mostrou oclusão do siste-

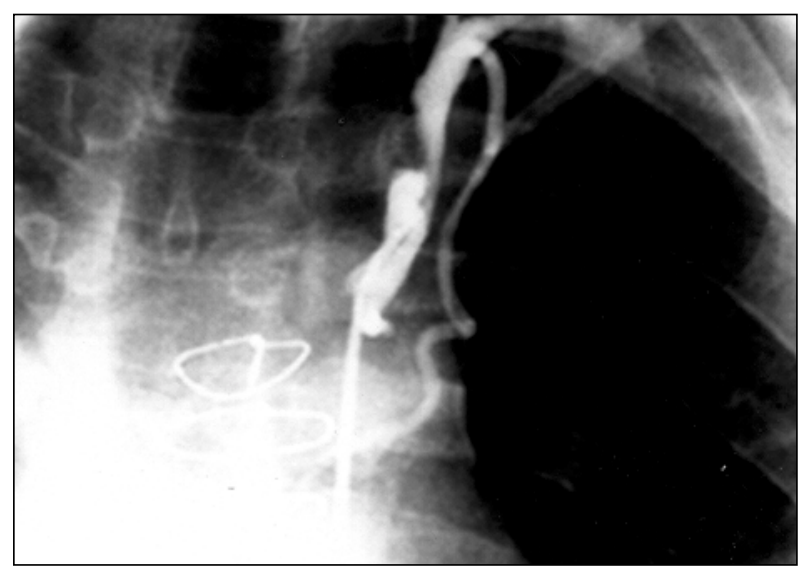

Fig. 1 - Angiografia em projeção ântero-posterior da artéria subclávia esquerda, evidenciando uma estenose severa no terço proximal antes da emergência da artéria mamária interna esquerda.

Instituto de Cardiologia do Rio Grande do Sul/Fundação Universitária de Cardiologia Correspondência: Rogério Sarmento-Leite - Unidade de Pesquisa do IC/FUC Av. Princesa Isabel, 395 - 90620-001 - Porto Alegre, RS

E-mail: pesquisa@cardnet.tche.br

Recebido para publicação em 26/11/01

Aceito em 6/5/02 ma coronariano nativo esquerdo. A MIE para ADA, e a safena para o ramo $\mathrm{Mg}$ encontravam-se pérvias; a safena para o ramo Dg estava ocluída e havia uma estenose ulcerada severa na porção proximal da artéria subclávia esquerda, antes da emergência da MIE (fig. 1). Realizou-se angioplastia com implante de stent periférico Jostent (Jomed, Rangendingen, Alemanha)6-12X38mm, expandido combalão Fox 10 x 40mm (Jomed, Beringen, Suíça) com 8 atmosferas de pressão com excelente resultado angiográfico final (fig. 2). Observou-se desaparecimento do gradiente de pressão entre os membros superiores e resolução dos sintomas do paciente. Estas imagens ilustram uma manifestação relativamente incomum, que deve necessariamente ser sempre lembrada, de sintomas anginosos limitantes e de fácil tratamento. Isto também reforça a importância de uma avaliação angiográfica das artérias subclávias antes de ser(em) utilizado(s) implante(s) de mamária quando uma CRM é planejada.

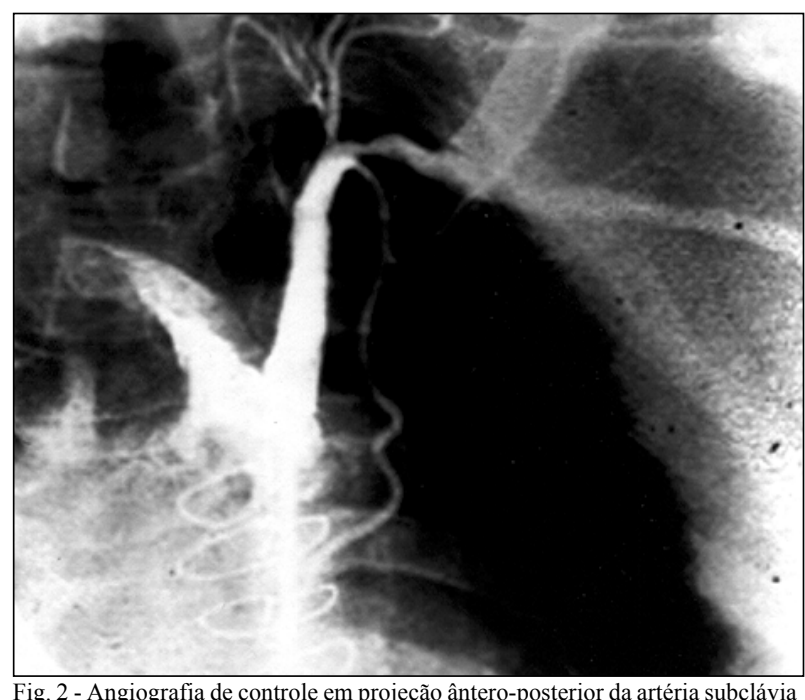
esquerda após a angioplastia com implante de stent. 


\section{Referências}

1. Saphira S, Braun SD, Puram B, et al. Percutaneous transluminal angioplasty of proximal subclavian artery stenosis after left internal mammary artery to left anterior descending artery bypass surgery. J Am Coll Cardiol 1991; 18: 1120-3.

2. Laub GW, Muralidharan S, Naidech H, et al. Percutaneous transluminal subclavian angioplasty in a patient with postoperative angina. Ann Thorac Surg 1991; 52: 850-1.
3. Salvadori Junior D, Ferreira BM, Gebara OC, et al. Treatment of subclavian artery stenosis with stents in patients previously treated with internal mammary left anterior descending artery bypass surgery. Arq Bras Cardiol 1997; 68: 363-5.

4. English JA, Carrel ES, Guidera SA, et al. Angiographic prevalence and clinical predictors of left subclavian stenosis in patients undergoing diagnostic cardiac catheterization. Catheter Cardiovasc Interv 2001; 54: 8-11. 